\title{
Out-Patient Pulmonary Rehabilitation Improves Medial-Lateral Balance in Subjects With Chronic Respiratory Disease: Proof-of-Concept Study
}

\author{
Michelle D Smith PhD, Elizabeth H Harvey MHSc, Wolbert van den Hoorn MSc, \\ Barbara L Shay PhD, Gisèle M Pereira BPT, and Paul W Hodges PhD
}

\begin{abstract}
BACKGROUND: Recent studies show balance impairment in subjects with chronic respiratory disease. The aim of this proof-of-concept study was to investigate clinical and quantitative measures of balance in people with chronic respiratory disease following participation in an out-patient pulmonary rehabilitation (PR) program to better understand features of balance improvement. A secondary aim was to probe possible mechanisms for balance improvement to provide the foundation for optimal design of future studies. METHODS: Eleven individuals with chronic respiratory disease enrolled in an 8-week out-patient PR program participated. Standing balance, measured with a force plate, in the medial-lateral and anterior-posterior directions with eyes open and closed was assessed with linear (SD and sway path length) and non-linear (diffusion analysis) center-of-pressure measures. Balance was evaluated clinically with the Timed Up and Go and Four Square Step Test. Fear of falling and balance confidence were assessed with questionnaires. RESULTS: After participation in PR, medial-lateral sway path length decreased $(P=.031)$, and center-of-pressure diffusion in the medial-lateral direction was slower $(P=.02)$ and traveled over less distance $(P=.03)$ with eyes closed. This suggests greater control of medial-lateral sway. There was no change in anterior-posterior balance $(P>.067)$. Performance improved on the Timed Up and Go (median [interquartile range] pre-PR $=9.4$ [7.9-12.8] vs post$\mathrm{PR}=8.1[7.3-12.2] \mathrm{s}, P=\mathbf{0 0 3}$ ) and Four Square Step Test (median [interquartile range] pre-PR $=9.3$ $[7.2-14.2]$ vs post-PR $=8.7[7.4-10.2] \mathrm{s}, P=.050)$. There were no changes in balance confidence $(P=.72)$ or fear of falling $(P=.57)$. CONCLUSIONS: Participation in an 8-week out-patient PR program improved balance, as assessed by clinical and laboratory measures. Detailed analysis of force plate measures demonstrated improvements primarily with respect to medial-lateral balance control. These data provide a basis for the development of larger scale studies to investigate the mechanisms for medial-lateral balance improvements following PR and to determine how PR may be refined to enhance balance outcomes in this population. (ClinicalTrials.gov registration NCT00864084.) Key words: chronic respiratory disease; pulmonary rehabilitation; exercise; balance. [Respir Care 2016;61(4):510-520. ( 2016 Daedalus Enterprises]
\end{abstract}

\section{Introduction}

Chronic respiratory diseases, such as COPD and asthma, are debilitating conditions that commonly lead to deterio-

Dr Smith, Mr van den Hoorn, and Dr Hodges are affiliated with the School of Health and Rehabilitation Sciences, The University of Queensland, St. Lucia, Queensland, Australia. Ms Harvey, Dr Shay, and Ms Pereira are affiliated with the School of Medical Rehabilitation, University of Manitoba, Winnipeg, Manitoba, Canada.

This project was supported by a research grant from The University of Queensland. Dr Hodges was supported by a Senior Principal Research ration of physical function, reduced quality of life, and increased dependence on medical services. Among the many well-established problems associated with COPD,

Fellowship from the National Health and Medical Research Council of Australia. The authors have disclosed no conflicts of interest.

Correspondence: Michelle D Smith PhD, School of Health and Rehabilitation Sciences, The University of Queensland, St Lucia, Queensland 4072, Australia. E-mail: m.smith5@uq.edu.au.

DOI: $10.4187 /$ respcare.04109 


\section{Out-Patient PR and Balance in Chronic Respiratory Disease}

compromised balance performance has been highlighted. ${ }^{1,2}$ Balance impairments in this group are greater in the medial-lateral (sideways) than in the anterior-posterior (forward-backward) direction, ${ }^{2}$ which is likely to have functional relevance because poor medial-lateral balance control has been linked to falls in older adults. ${ }^{3,4}$ It is not surprising that preliminary evidence shows a relationship between compromised balance and increased incidence of falls in people with respiratory disease. ${ }^{5}$

As identified for other populations, ${ }^{6}$ rehabilitation of balance is likely to reduce fall risk in people with respiratory disease. Pulmonary rehabilitation (PR) programs, which typically include cardiovascular endurance training and upper and lower limb muscle strengthening, are common interventions for this population. ${ }^{7}$ Although typical PR programs do not specifically target balance performance, they address a range of features that have been associated with deficits in balance, such as lower extremity weakness, ${ }^{8}$ decreased exercise tolerance and physical activity, ${ }^{9,10}$ and dyspnea. ${ }^{11}$

Access to out-patient PR programs is increasing, and it is timely to determine whether the current content of these programs improves balance, as has been shown for more intensive in-patient PR programs, ${ }^{12,13}$ or whether refinement of the program content is necessary. Further, although balance quality is related to falls, it is necessary to directly investigate whether improved balance translates to reduced fall risk. This has not been tested for any type of PR program. Recent studies have found small improvements in clinical measures of balance (as a surrogate for fall risk) after participation in a 6-week intensive in-patient PR program ${ }^{13}$ and further improvements in balance when specific balance training is added. ${ }^{12}$ A limitation of the existing work is the reliance on clinical balance measures. Although clinical measures are important, they do not provide insight into the parameters of balance function that underpin compromised balance and fall risk or the features improved by PR. Quantitative analysis of balance parameters, such as those related to movement of the center of pressure, enable detailed analysis of the specific features of balance that may be compromised and improved with training. Most balance research focuses on linear components of center of pressure (eg, SD and mean). Although these provide some insight into mechanisms underlying potential balance improvements, nonlinear measures, such as diffusion analysis, provide greater detail about how balance is controlled and changes over time. ${ }^{14}$ Because linear and nonlinear measures have not been used to investigate balance in people with respiratory disease, preliminary work is required to ascertain the most meaningful parameters and provide baseline data for issues such as sample size calculation for randomized controlled trials to test the effect of out-patient PR programs on balance and fall incidence.

\section{QUICK LOOK}

\section{Current knowledge}

People with COPD have impaired balance, particularly in the medial-lateral direction, and an increased incidence of falls. In-patient pulmonary rehabilitation programs have been shown to improve clinical measures of balance. Although important, these measures do not provide insight into the parameters of balance that are improved following pulmonary rehabilitation.

\section{What this paper contributes to our knowledge}

Clinical and force plate measures of balance improved after participation in an 8-week out-patient pulmonary rehabilitation program. Detailed analyses revealed that balance improvements were primarily due to improvements in medial-lateral balance control. These findings provide the foundation for the development of randomized controlled trials to investigate the effect of pulmonary rehabilitation on reducing fall incidence.

As an initial step toward design of a randomized controlled trial of the effect of out-patient PR programs on balance and fall incidence, the objective of this proof-ofconcept study was to provide data to resolve several key issues that would provide the foundation for a large trial design. Our primary aim was to determine whether outpatient PR improves different features of balance, functional balance (as measured by clinical balance measures) and balance in quiet standing (using linear and non-linear quantitative measures), as a surrogate measure of fall risk. Our secondary aim was to investigate changes in measures of strength and psychological characteristics, such as balance confidence and fear of falling, to provide initial insight into possible mechanisms for balance improvement that may be appropriate to investigate in larger trials.

\section{Methods}

\section{Subjects}

Eleven individuals (4 males, 7 females; demographic details in Table 1) with physician-diagnosed stable chronic respiratory disease who planned to commence an out-patient PR program participated in this proof-of-concept study. Participants were diagnosed with COPD $(n=9$, including 3 with a primary diagnosis of bronchitis/bronchiectasis) and asthma $(n=2)$. Inclusion criteria were clinical diagnosis of respiratory disease provide by the treating physician, commitment to commence and regularly attend the PR program, and no change in medication 
Table 1. Baseline Demographic Data

\begin{tabular}{lc}
\hline \hline \multicolumn{1}{c}{ Variables } & Values \\
\hline Female sex, $n(\%)$ & $64(7)$ \\
Age, mean $\pm \mathrm{SD}$ y & $70 \pm 8$ \\
Body mass index, mean $\pm \mathrm{SD} \mathrm{kg} / \mathrm{m}^{2}$ & $29.4 \pm 11.9$ \\
$\mathrm{FEV}_{1}$, mean $\pm \mathrm{SD} \mathrm{L}$ & $1.52 \pm 0.77$ \\
$\mathrm{FEV}_{1}$, mean $\pm \mathrm{SD} \%$ predicted & $60.1 \pm 29.1$ \\
$\mathrm{FEV}_{1} / \mathrm{FVC}$, mean $\pm \mathrm{SD}$ & $0.54 \pm 0.19$ \\
$\mathrm{PEF}$, mean $\pm \mathrm{SD} \mathrm{L}$ & $234.2 \pm 114.9$ \\
$\mathrm{PEF}$, mean $\pm \mathrm{SD} \%$ predicted & $64.1 \pm 31.9$ \\
$\mathrm{SGRQ}$ score, mean $\pm \mathrm{SD}$ & $47.6 \pm 9.3$ \\
$\mathrm{IPAQ}$ score, mean $\pm \mathrm{SD}$ & $2,817 \pm 1,771$ \\
Frenchay Activity Index score, mean $\pm \mathrm{SD}$ & $67.2 \pm 16.8$ \\
& \\
\hline PEF $=$ peak expiratory flow & \\
SGRQ $=$ St George Respiratory Questionnaire & \\
IPAQ $=$ International Physical Activity Questionnaire & \\
\hline
\end{tabular}

for the last 2 months. Patients were excluded if they had unstable cardiac disease, neurological conditions, or musculoskeletal conditions that prevented participation in the PR program or testing sessions.

Three further subjects (2 males, 1 female; mean age $55 \mathrm{y}$; body mass index $28.2 \mathrm{~kg} / \mathrm{m}^{2} ; \%$ predicted $\mathrm{FEV}_{1}$ 52\%; \% predicted PEF 56\%) volunteered for this study but withdrew from the PR program before the initial $(n=2)$ or final ( $n=1)$ testing sessions. Ethical approval for this study was obtained from the institutional research ethics committee, and all subjects provided informed consent.

\section{Procedures}

Individuals attending PR were invited to complete testing and questionnaires before initiation and after completion of the 8-week out-patient PR program. Investigators (MDS and $\mathrm{EHH}$ ) who were not involved in client care or implementation of the PR program collected all data. Results of the initial testing sessions were not known to investigators and subjects at the time of final testing, and all data were analyzed upon completion of the study. Questionnaires were completed first, followed by measures of balance and strength, which were performed in a random order. Subjects were instructed to rest as needed to avoid the effect of acute changes in breathlessness on test results. ${ }^{2,15}$

\section{Measures of Balance}

Ground reaction forces were measured using a force plate (AMTI, Watertown, Massachusetts) with a sampling rate of 100 samples/s. Center of pressure was calculated using standard formulas. Subjects stood on the force plate in relaxed stance with feet placed hip width apart. Trials were recorded for $60 \mathrm{~s}$ with eyes open and closed.

Clinical measures of balance involved the Timed Up and Go and Four Square Step Test. For the Timed Up and Go, the time taken for the participant to stand from a chair, walk $3 \mathrm{~m}$, turn around, and return to the chair was recorded. ${ }^{16}$ Subjects were asked to do this as quickly and safely as possible. High test-retest reliability of the Timed Up and Go has been reported in older community-dwelling individuals. ${ }^{17}$ The Timed Up and Go has been used in previous balance research in individuals with COPD. ${ }^{13}$ In the Four Square Step Test, subjects stepped to 4 corners of a square in a clockwise and then counterclockwise direction as quickly as possible. ${ }^{18}$ The time taken to complete this circuit was recorded. This test has high inter-rater and test-retest reliability. ${ }^{18}$ It was included because it involves a greater challenge to balance in the medial-lateral direction than the Timed Up and Go, and this direction is considered critical for fall risk ${ }^{3,4}$ and the greatest compromise in this clinical group. ${ }^{2}$

\section{Quadricep Muscle Strength}

Maximum voluntary isometric quadricep muscle strength of the right leg was measured using a KinCom AP 125 (Isokinetic International, East Ridge, Tennessee). Subjects sat with the hip and knee flexed to $90^{\circ}$ and were asked to push as hard as possible to extend the knee isometrically against resistance.

\section{Questionnaires}

Subjects completed a short baseline questionnaire to obtain information about demographics (ie, age, height, and weight), physical activity participation (using the International Physical Activity Questionnaire ${ }^{19}$ and the Frenchay Activities Index ${ }^{20}$ ), and health-related quality of life (using the St George Respiratory Questionnaire ${ }^{21,22}$ ). These measures were recorded to characterize the study population.

The Falls Efficacy Scale-International and the Activities-Specific Balance Confidence scale were completed before and after participation in the PR program to assess changes in fear of falling and balance confidence. The Falls Efficacy Scale-International assesses fear of falling during 16 common physical and social activities, such as cleaning the house and ascending and descending stairs. ${ }^{23}$ Answers range from not at all concerned to very concerned on a 4-point scale, and the total score was calculated as the average response. This questionnaire has excellent internal and test-retest reliability. ${ }^{23}$ The Activities-Specific Balance Confidence scale measures balance confidence during 16 activities of progressive difficulty, such as going up and down stairs, 


\section{Out-Patient PR and Balance in Chronic Respiratory Disease}

reaching for objects, and walking in crowded areas. ${ }^{24}$ Confidence in performing an activity without losing balance is rated on an 11-point scale ranging from $0 \%$ (no confidence) to $100 \%$ (completely confident). Scores for each item were averaged. This questionnaire is sensitive to detect changes in function following rehabilitation $^{25}$ with proven internal consistency and test-retest reliability. ${ }^{24}$

\section{Intervention}

The 8-week PR program involved customized exercise prescription directed by a physiotherapist. Subjects also attended weekly educational sessions on topics such as the importance of exercise, medication use, energy conservation, pacing, nutrition, coping skills, and breathing exercises. At the start of the PR program, a physiotherapist assessed each participant to determine individual exercise goals, ability, and tolerance. Subjects were prescribed an individualized exercise program to be performed 1-2 times/week with physiotherapist supervision and 3-5 sessions/week of independent home exercise. Exercises aimed to improve cardiovascular fitness, upper and lower limb strength and endurance, and general flexibility.

All subjects were given a walking program to improve cardiovascular fitness. The distance and time subjects were asked to walk was determined from the results of a 6-min walk test performed during the initial assessment. ${ }^{26}$ Subjects with access to exercise facilities or equipment were also prescribed cardiovascular exercise, which included cycling on a stationary bike $(n=4)$ and aquatic $(n=1)$ or land-based $(n=2)$ exercise classes. Subjects were encouraged to perform cardiovascular exercises 5-6 times/week. Exercises were progressed by increasing exercise distance, time, speed, or incline (treadmill-based programs only).

Subjects were prescribed exercises to improve muscular strength/endurance of major muscle groups of the upper (ie, biceps, triceps, deltoid, rhomboids, pectoralis major, and trapezius muscles) and lower (ie, quadricep, hamstring, gluteal, hip abductor, gastrocnemius, and soleus muscles) limbs. Exercises were performed in both sitting and standing positions. Free weights, body weight, and elastic tubing were used for resistance. Resistance type depended on the participant's preference and the muscle group. Resistance was determined by the subjects' ability to complete $8-12$ repetitions of the exercise with correct technique. Subjects were encouraged to complete $1-2$ sets of $8-12$ repetitions maximum of each strengthening exercise 4 times/week. Exercises were progressed by increasing resistance and sets (1-2 sets).

Daily stretching exercises were prescribed to improve general flexibility. Static stretches were used for major muscle groups of the neck, trunk, lower limbs, and upper limbs. Subjects were instructed to hold the stretch for ap- proximately $20 \mathrm{~s}$ and repeat each stretch twice. These exercises were not progressed in the PR program. Subjects completed an exercise diary to document the type and amount of exercise performed each day. Exercise diaries were returned to investigators weekly.

\section{Data Analysis}

The Timed Up and Go, Four Square Step Test, and quadricep strength test were repeated 3 times, and the average was used for analysis. Force plate data were analyzed using Matlab (R2012a, Mathworks, Natick, Massachusetts). Data were low pass-filtered with a bidirectional 4th order Butterworth filter $(15 \mathrm{~Hz})$ and analyzed using both simple linear measures (as is common in this field) and non-linear diffusion analysis, which provides detail of structure of the signal and how this changes over time. Diffusion analysis is considered more sensitive to detect differences in quality of balance than the standard linear measures. ${ }^{14}$ For the linear measures, medial-lateral and anterior-posterior center-of-pressure displacement was quantified as the SD of center of pressure (ie, average displacement from the mean center-of-pressure position) and length of the center-of-pressure path/s (sum of absolute distance traveled divided by $60 \mathrm{~s}$; sway path length).

Diffusion analysis comes from Brownian motion ${ }^{27}$ and can be explained with the following analogy. Diffusion is the rate at which, for example, a drop of food coloring disperses (diffuses) over a volume of water. In terms of balance control, the center of pressure diffuses over the area of support. However, because the area of support has a boundary, diffusion of center of pressure cannot continue at the same rate in one direction; otherwise, a fall would occur. For example, the center of pressure of a person can move in one direction at a certain speed until the moment when center of pressure is corrected to prevent a fall. At this point, diffusion rate will be lower and with a more corrective nature. The center of pressure moves faster at some times than at others. In contrast to linear measures of center-of-pressure displacement, diffusion analysis takes into account these different temporal aspects of balance control. We considered this to be important, because it might provide insight into specific features of balance control improved by PR.

Several measures can be extracted from diffusion analysis that provide insight into the quality of balance control. To calculate diffusion of center of pressure in the anteriorposterior and medial-lateral directions, the average squared distance between each point separated in time from 0.01 to $10 \mathrm{~s}$ was determined and plotted against time. Figure 1 displays an example of diffusion analysis for a representative subject. ${ }^{14}$ The short-term region of the plot was defined as the linear fit with a correlation of $>0.995$ (slope of regression line fitted to the short-term region; short- 


\section{Out-Patient PR and Balance in Chronic Respiratory Disease}
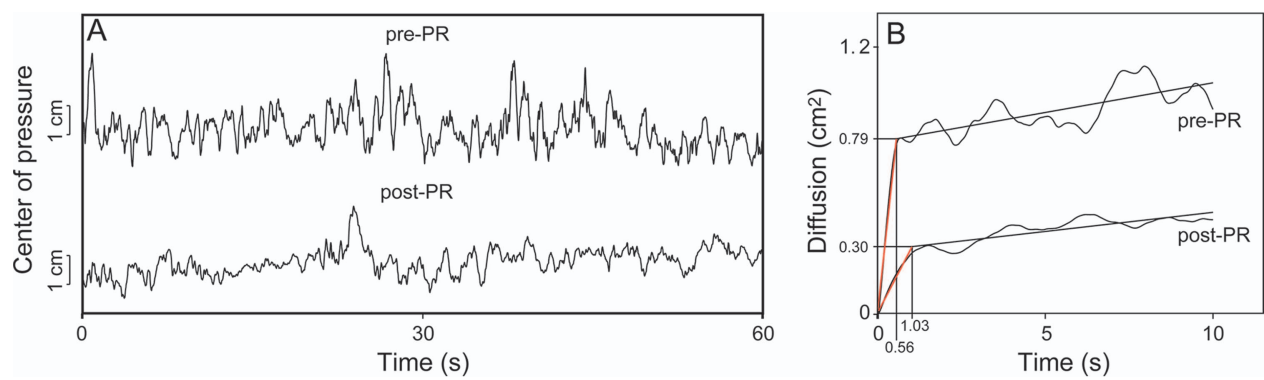

Fig. 1. Center of pressure in the medial-lateral direction $(A)$ and the corresponding diffusion analysis (B) for pre- and post-pulmonary rehabilitation (PR) of a representative participant. The diffusion plots (B) show 2 parts in the graph and are related to the behavior of the center of pressure over the short and long term. The 2 parts are determined by the intersection of the regressions of the short-term (red) and long-term (black; see methods section for more details) data. The slope (diffusion coefficient [Ds]) of the short-term regression line relates to the rate of diffusion $\left(\mathrm{cm}^{2} / \mathrm{s}\right)$ and is higher pre-PR $\left(0.69 \mathrm{~cm}^{2} / \mathrm{s}\right)$ than post-PR $\left(0.14 \mathrm{~cm}^{2} / \mathrm{s}\right)$. In contrast to the long-term region, short-term balance control is not corrective; an increase in the center of pressure is usually followed by another increase (persistent, exponent > 1). After PR, subjects moved less far and slower before corrective balance was initiated in the medial-lateral direction than before PR. This finding can be visually observed in the center-of-pressure signals (A), where the center of pressure moves faster (Ds) and farther pre-PR than post-PR. We interpret this as an improvement in balance control.

term diffusion coefficient), and the long-term region was defined as the linear fit of the remainder of the diffusion plot (slope of regression line fitted to the long-term region; long-term diffusion coefficient). ${ }^{28}$ Diffusion and the time of the diffusion plot were also log-transformed (power law, exponent) to assess whether center of pressure had a corrective nature. If the slope of the linear fit of the shortor long-term regions is $>1$, then the nature of balance control is not corrective (ie, an increase in center of pressure is usually followed by another increase in center-ofpressure displacement). If the slope is $<1$, then balance control has a corrective nature (ie, an increase is usually followed by a decrease in center-of-pressure displacement). The intersection of the linear fits of the short and longterm regions of the diffusion plot gives information about how long (critical point in time) and how far (critical point in distance) subjects allowed center of pressure to diffuse in the short term and when subjects changed their nature of balance control. ${ }^{14}$ In general, more optimal balance control is characterized by (i) slower (less fast) diffusion and (ii) less diffusion distance before correction.

\section{Statistical Analysis}

Tests for normality indicated that quadricep strength data were normally distributed, but Timed Up and Go and Four Square Step Test data were not. Ordinal data (balance confidence and fear of falling) and the non-normally distributed Timed Up and Go and Four Square Step Test data were compared between measures made before and after participation in PR using the Wilcoxon matched-pairs signed-ranks test. Quadricep strength measures were compared before and after PR using a paired $t$ test. Linear and non-linear measures of balance were compared with repeated measures analysis of variance with 2 repeated mea- sures: time (pre-PR vs post-PR) and eye condition (open vs closed). Because data were not normally distributed, balance measures were log-transformed. Analyses were performed with Statistica 7 (StatSoft, Tulsa, Oklahoma) with statistical significance set at $P<.05$. Duncan's multiple range test was used for post hoc testing. Data are presented as mean $\pm \mathrm{SD}$ for normally distributed variables and median (interquartile range) for ordinal and non-normally distributed variables throughout.

\section{Results}

\section{Participation in PR}

Subjects attended the PR program $9.3 \pm 5.1$ times over the 8-week period, or $1.3 \pm 0.5$ times/week for exercise sessions, with one additional attendance each week for group educational sessions. Exercise diaries were completed and returned for 8 of the 11 subjects (73\%). According to the completed exercise diaries, subjects exercised $6.0 \pm 1.0 \mathrm{~d} /$ week of combined in-program and home exercise throughout the 8-week PR program.

\section{Clinical Measures of Balance, Quadricep Muscle Strength, and Questionnaires}

After participation in the PR program, subjects' performance improved on the Timed Up and Go and Four Square Step Test (see Table 2 for median [interquartile range] and $P$ values). Quadricep muscle strength did not change with PR (see Table 2). There was no change in balance confidence (Activities-Specific Balance Confidence) or fear of falling (Falls Efficacy Scale-International) after PR (see Table 2). 
Out-Patient PR and Balance in Chronic Respiratory Disease

Table 2. Functional Balance Tests and Questionnaires Before and After Participation in Pulmonary Rehabilitation

\begin{tabular}{|c|c|c|c|}
\hline & Pre-PR & Post-PR & $P$ \\
\hline TUG score, median (IQR) s & $9.4(7.9-12.8)$ & $8.1(7.3-12.2)$ & .003 \\
\hline FSST score, median (IQR) s & $9.3(7.2-14.2)$ & $8.7(7.4-10.2)$ & .050 \\
\hline $\begin{array}{l}\text { Quadriceps strength, mean }( \pm \mathrm{SD} \text {, range }) \\
\text { newton-meters }\end{array}$ & $72(28,36-124)$ & $76(27,35-124)$ & .37 \\
\hline ABC scale, median (IQR) & $74(59-98)$ & $73(55-96)$ & .72 \\
\hline FESI scale, median (IQR) & $1.8(1.2-2.3)$ & $1.9(1.2-2.1)$ & .54 \\
\hline $\begin{array}{l}\mathrm{PR}=\text { pulmonary rehabilitation } \\
\text { TUG = Timed Up and Go } \\
\text { FSST = Four Square Step Test } \\
\text { IQR = interquartile range } \\
\text { ABC = Activities-Specific Balance Confidence } \\
\text { FESI = Falls Efficacy Scale-International }\end{array}$ & & & \\
\hline
\end{tabular}

\section{Linear Measures of Balance}

Only one linear measure of center-of-pressure displacement improved following participation in PR. When subjects stood with their eyes closed, sway path length was shorter in the medial-lateral direction after PR than before PR (see Fig. 2 and Table 3 for $P$ values). SD of anteriorposterior or medial-lateral center-of-pressure movement or anterior-posterior sway path length was not changed by participation in PR (see Fig. 2 and Table 3). As expected, the SD and sway path length of anterior-posterior centerof-pressure motion and sway path length in the anteriorposterior and medial-lateral directions were higher with eyes closed than open (see Fig. 2 and Table 3). Sway path length in the medial-lateral direction was longer with eyes open than closed but only before PR. A similar trend was present for SD of medial-lateral center-of-pressure motion, but this did not reach significance (main effect: eye condition, $P=.067$ ).

\section{Non-Linear Measures of Balance}

After PR, short-term center-of-pressure diffusion in the medial-lateral direction was slower (short-term diffusion) and over less distance (critical point in distance) than before PR (see Fig. 2 and Table 3). This was only observed when subjects stood with their eyes closed. This is illustrated by data for a representative participant in Figure 1. Long-term diffusion and critical point in time in the medial-lateral direction were not affected by PR. Diffusion analysis of the center of pressure in the anterior-posterior direction did not differ between measures made before and after PR (see Fig. 2 and Table 3). In both the anteriorposterior and medial-lateral directions, the exponent of the short-term diffusion region was $>1$, and the exponent of the long-term diffusion region was $<1$. This reflects noncorrective behavior in the short term and corrective behavior in the long term. PR did not affect the exponents of the short- and long-term diffusion regions in anterior-posterior and medial-lateral directions. As expected, based on task difficulty, the anterior-posterior short-term diffusion was faster, shorter (critical point in time), and farther (critical point in distance) when standing with eyes closed than eyes open (see Fig. 2 and Table 3). In addition, the exponent of long-term diffusion in the anterior-posterior direction was lower with eyes closed than open. This suggests increased control in the long-term potentially due to greater and farther diffusion in the short term with eyes closed than with eyes open. In the medial-lateral direction, short-term diffusion was faster and farther (critical point in distance) with eyes closed but only pre-PR (see Fig. 2 and Table 3 ). The long-term diffusion rate did not differ between the eyes open and closed conditions (see Fig. 2 and Table 3).

\section{Discussion}

Findings from this proof-of-concept study demonstrate that participation in an 8-week out-patient PR program that did not specifically target balance improved some aspects of balance in people with chronic respiratory disease. Although improvements were identified across the 3 domains of balance measurement (clinical tests of balance and both linear and non-linear measures of center-ofpressure displacement), improvements generally involved the medial-lateral direction. Participation in PR not only decreased the total amount of medial-lateral center-ofpressure displacement; the non-linear measures also revealed improvements in how quickly individuals with respiratory disease were able to correct postural sway in the medial-lateral direction. Taken together with the importance of medial-lateral balance for fall risk, these data provide the foundation to test whether balance changes after PR translate to meaningful changes in fall incidence in this group. These data also provide the basis to 

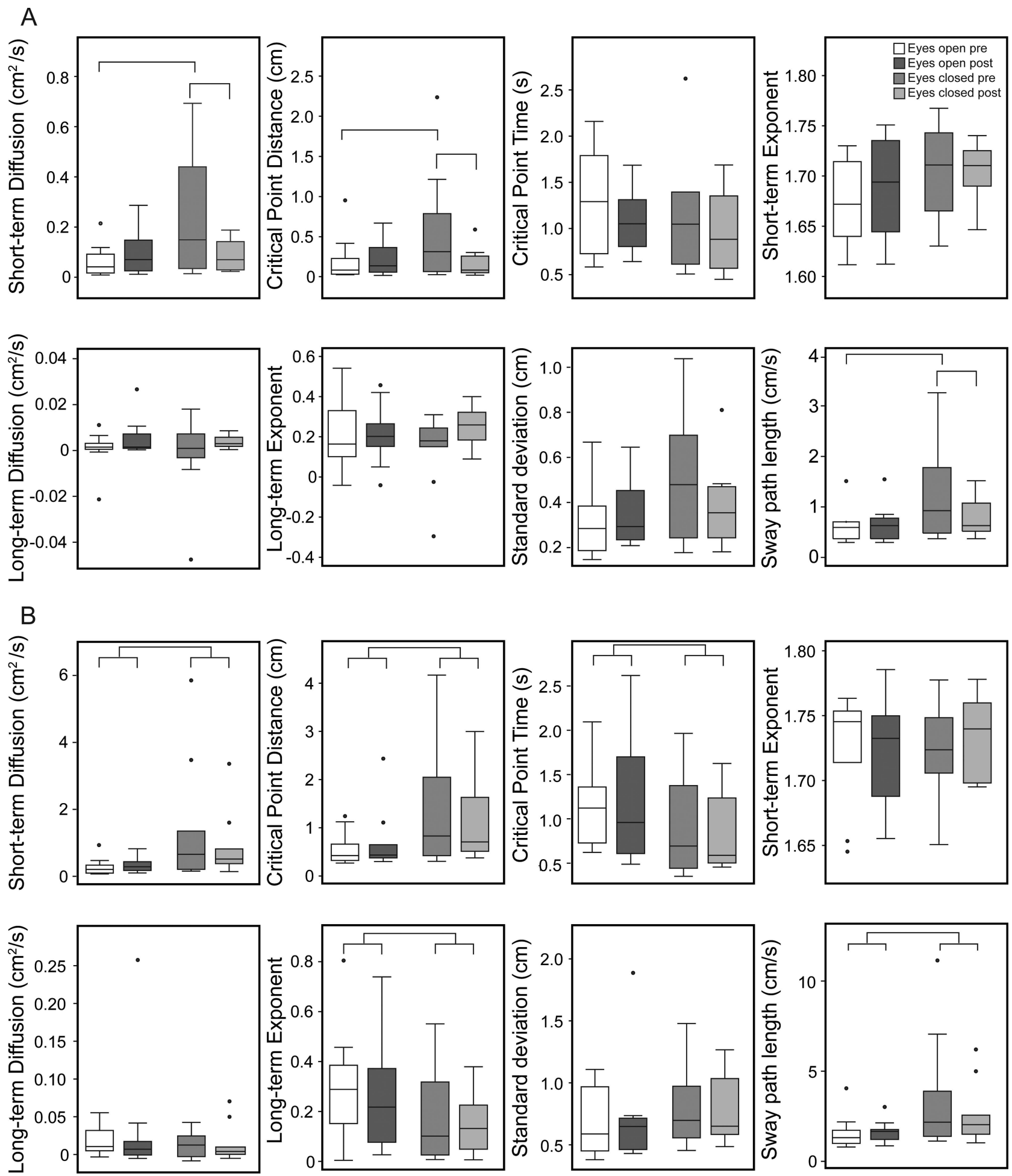

Fig. 2. Linear and non-linear outcome measures of standing balance in the medial-lateral $(A)$ and anterior-posterior (B) direction when participants stood on the force plate for $60 \mathrm{~s}$ with eyes open and eyes closed, pre- and post-pulmonary rehabilitation. Brackets highlight significant differences between pre- and post-pulmonary rehabilitation and between eyes open and closed $(P<.05)$. Boxes denote median and upper and lower interquartile ranges, whiskers show upper and lower adjacent values, and dots represent values outside this range. 
Table 3. Results of Repeated Measures Analysis of Variance of Linear Measures and Diffusion Analysis of Center-of-Pressure Data With Eyes Open and Closed Before and After Participation in Pulmonary Rehabilitation

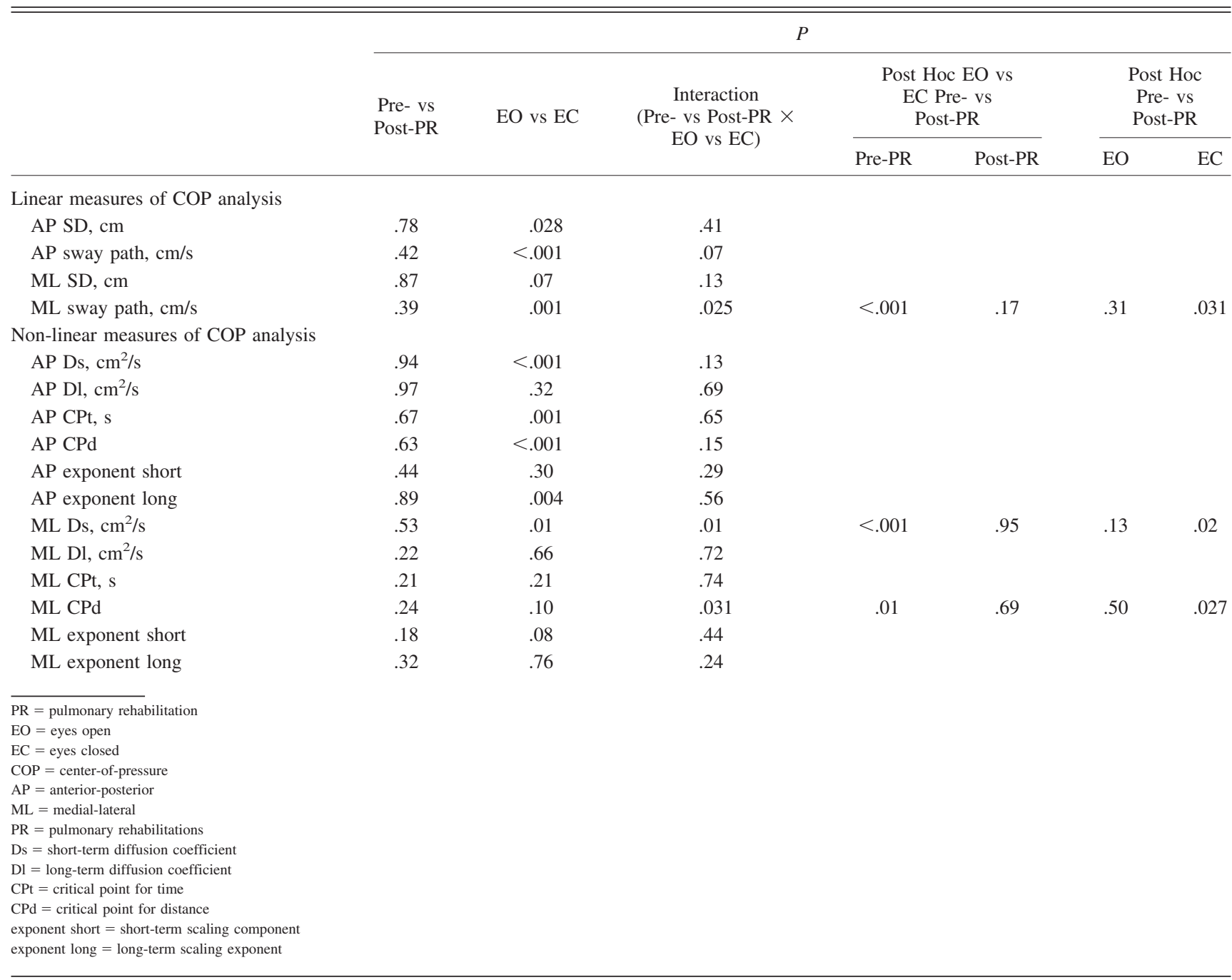

consider measures to include in a larger trial to study underlying balance mechanisms.

\section{Clinical and Non-Linear Quantitative Measures of Balance Improved With PR}

This is the first study in individuals with chronic respiratory disease to use quantitative analysis of medial-lateral and anterior-posterior center-of-pressure balance parameters. These measures provide detailed information regarding the specific aspect of balance that was improved (ie, medial-lateral balance control) following participation in an out-patient PR program. In light of the improvements in medial-lateral balance control (which has been shown to be deficient in people with $\mathrm{COPD}^{2}$ ) following PR and the relationship between medial-lateral balance and risk of falls, ${ }^{3,4}$ inclusion of these detailed measures of medial- lateral balance in future randomized controlled trials to prospectively investigate the effect of PR on balance and falls is likely to yield important information about the potential mechanisms. Clear understanding of the mechanisms for balance improvement may help guide identification of modifiable targets to further refine and enhance treatment efficacy.

Similar to what was found in previous research of intensive in-patient PR programs, out-patient PR improved clinical measures of balance. The average improvement on the Timed Up and Go of $1.4 \mathrm{~s}$ in our study is similar to that reported by Beauchamp et al. ${ }^{13}$ (1.5 s) following a 6-week in-patient PR. Although balance studies in people with respiratory disease have not used the Four Square Step Test, the average improvement in Four Square Step Test score (1.9 s) was of a similar order of magnitude to that in the Timed Up and Go. The Four Square Step Test has the 


\section{Out-Patient PR and Balance in Chronic Respiratory Disease}

advantage of challenging patients in the medial-lateral direction, which is the direction of balance impairment observed in this population ${ }^{2}$ and the direction of balance impairment linked to falls in older adults. ${ }^{3,4}$

\section{Linear and Non-Linear Quantitative Measures of Balance Improved in the Medial-Lateral Direction}

Analysis of center of pressure indicated that improvements were restricted to the medial-lateral components of balance and were only found when subjects stood with their eyes closed. Standard linear measures showed less center-of-pressure movement in the medial-lateral direction (ie, shorter sway path length) after PR than before PR. This was substantiated and extended by results from the non-linear diffusion analysis in this study. Subjects moved faster and farther in the medial-lateral direction before correcting to maintain upright balance before PR than after $\mathrm{PR}$, suggesting that PR positively influenced short-term medial-lateral balance control. This improved quality of medial-lateral balance is likely to be meaningful because people with COPD have poorer control of balance in the medial-lateral direction than age and gender-matched control subjects. ${ }^{2}$

There are several potential mechanisms to explain the improved control of medial-lateral balance in people with respiratory disease following PR. Control of medial-lateral balance is more dependent on coordinated movements of trunk and hip than anterior-posterior balance. This is because the ankles (which are critical for anterior-posterior control) make a limited contribution to balance control in the medial-lateral direction. ${ }^{29,30}$ Thus, improved mediallateral balance after PR could be explained by enhanced contribution of the hip and/or trunk to balance. Several elements of the PR program could explain this improvement. First, PR exercises targeted strength and endurance of the hip and trunk muscles, and this could improve their contribution to medial-lateral balance control. Because hip and trunk muscle strength was not formally assessed, we cannot comment on the effect of PR on these measures, and further investigation is warranted. Second, the increased contribution of the hip and trunk to balance might be explained by improved dyspnea and changes in breathing efficiency. Trunk muscles are required for both respiration and trunk motion/stability. ${ }^{31}$ Greater activation of trunk muscles for breathing in respiratory disease to address airway resistance has been proposed to reduce the ability of the trunk to participate in balance reactions. ${ }^{32}$ Improved respiratory function after PR may optimize respiratory activity of trunk muscles and enable greater contribution of the trunk to the control of medial-lateral balance. ${ }^{33}$ Third, increased physical activity as a result of participation in PR may enhance sensory function of body movement and position (ie, proprioception) with direct benefit for inter- pretation and response to movement of the center of pressure. These potential mechanisms can be specifically addressed in prospective studies of balance and falls by inclusion of detailed measures.

\section{Clinical and Quantitative Measures of Balance}

The relationship between clinical and laboratory measures of balance requires consideration in future research. Because the Four Square Step Test involves a greater emphasis on medial-lateral movements than the Timed Up and Go test, improvements in medial-lateral balance control may be more related to improvements in the Four Square Step Test. Although further research into the relationship between clinical (Timed Up and Go and Four Square Step Tests) and quantitative measures of balance is needed, data suggest that both of these measures are likely to provide meaningful data in future trials and clinical assessments to understand the underlying mechanisms of PR on balance and falls.

Changes to medial-lateral control of balance were only revealed when subjects moved dynamically (clinical measures) and when they stood with their eyes closed, not eyes open. This suggests that standing with the eyes open provides insufficient challenge to reveal improvements in balance control in people with respiratory disease. The Timed Up and Go and Four Square Step Test assess complex dynamic balance as well as other aspects of fitness, including exercise capacity, dyspnea, muscular endurance, and motor planning. Changes in several of these fitness parameters may also improve non-linear measures of balance.

\section{Balance Confidence, Fear of Falling, and Quadricep Strength}

The finding that balance confidence did not change following participation in the PR program concurs with other work that found no improvement in this measure following a 6-week in-patient PR program ${ }^{13}$ and a PR program that included specific balance training. ${ }^{12}$ Taken together, these findings imply that PR with and without balancespecific exercises is not sufficient to address balance confidence. This may require specific attention in the design of future programs. Fear of falling post-PR has not previously been investigated. Our data suggest that, similar to balance confidence, fear of falling may not change following PR. Eight weeks may not be sufficient time to change these aspects. Improvements in balance confidence and fear of falling have been identified in older adults with a history of falls after participation for 8 months, but not 4 months, of intense balance-specific (ie, Tai Chi) training. ${ }^{34}$ This suggests that even adults who have fallen previously and have considerably lower balance confidence at base- 


\section{Out-Patient PR and Balance in Chronic Respiratory Disease}

line compared with our subjects (mean [SD] 53.5 [9.2] vs 75.3 [20.3]) require months of balance training to improve balance confidence and fear of falling. ${ }^{34}$ Alternatively, it is also possible that confidence and fear may require specific attention with approaches such as cognitive behavioral therapy. 35,36

Isometric quadricep muscle strength also did not change following PR in this study. This is perhaps not surprising because subjects used an 8-12 repetition maximum resistance for quadricep exercises, which is less than the $80 \%$ of 1-repetition maximum recommended to improve strength in untrained individuals. ${ }^{37}$ Lower limb muscle strength has been linked to increased risk of falls, ${ }^{38}$ and thigh muscle strength and endurance are decreased in people with respiratory disease. ${ }^{39}$ Because the quadricep and hamstring muscles generate force in the anterior-posterior plane, they have limited capacity to control balance in the mediallateral direction. Muscles acting on the hip have a greater contribution to medial-lateral balance control. Considering our findings of medial-lateral control, future research should investigate hip muscle strength and endurance, particularly the abductor and adductor muscles, in people with respiratory disease.

\section{Methodological Considerations}

Interpretation of data from this study requires consideration of several aspects of the methodology. First, the study did not include a control group without participation in PR and subjects self-selected to be involved in the PR program. Participant numbers may lack sensitivity to detect small effects of PR. Data from this proof-of-concept study form the foundation for a study with a larger sample size, control group, and randomized design to draw more robust conclusions that could augment this preliminary exploratory finding. As indicated, this study was designed as a proof-of-concept study to determine whether an outpatient PR program can improve balance and to identify the measures (clinical and detailed quantitative measures) that would be likely to inform mechanistic understanding of the changes in balance to aid program refinement. This was undertaken as the initial step toward conducting larger-scale randomized controlled trials for the prospective assessment of falls. Second, study subjects received an individualized exercise program that involved consideration of participant preferences and equipment availability. Therefore, as is typical of most PR programs, the prescribed exercises were not standardized between subjects. Different exercise modes may have influenced study results and should be considered in interpretation of data. Third, the number of PR sessions attended varied between subjects. Some individuals exercised on their own, whereas others preferred to complete more of their exercise sessions at the PR facility. Despite differences in supervised and unsupervised exercise sessions, subjects all completed a similar number of exercise sessions each week (average of 6 exercise sessions/week [range 5-7 sessions/week]). Methods to improve adherence to exercise at the PR program, drawing on behavior change theory, are worthy of consideration. Finally, the study population was overweight, with an average body mass index of $29.4 \mathrm{~kg} / \mathrm{m}^{2}$. This may limit generalizability of the findings; however, body weight would be unlikely to affect study results due to the repeated measures design.

\section{Conclusions}

This study demonstrates that participation in an 8-week out-patient PR program improves balance. Detailed analysis of quantitative measures revealed that this is most likely explained by improved quality of medial-lateral balance. This observation is important for 2 reasons: First, poor control of medial-lateral balance has been associated with an increased risk of falls ${ }^{3,4}$, and second, the greatest deficit in balance in people with COPD is in the mediallateral direction. ${ }^{1,2}$ The findings have important implications for the design of future studies, including randomized controlled clinical trials to test the efficacy of PR to reduce fall incidence. This study provides the foundation to focus investigation of balance on medial-lateral parameters using clinical and quantitative measures, with a focus on more challenging balance tasks (eg, eyes closed; dynamic). The results highlight that understanding of the mechanisms for the effect of PR on falls could be enhanced by inclusion of non-linear balance measures and consideration of hip muscle strength. They also suggest that psychological factors relevant to falls (fear of falling and balance confidence) may not be sufficiently addressed in PR programs. Finally, the data provide values that can be used to estimate sample sizes required to address these issues in detail.

\section{ACKNOWLEDGMENTS}

We acknowledge the help of the Bachelor of Medical Rehabilitation in Physical Therapy students who assisted in running the PR program.

\section{REFERENCES}

1. Butcher SJ, Meshke JM, Sheppard MS. Reductions in functional balance, coordination, and mobility measures among patients with stable chronic obstructive pulmonary disease. J Cardiopulm Rehabil 2004;24(4):274-280.

2. Smith MD, Chang AT, Seale HE, Walsh JR, Hodges PW. Balance is impaired in people with chronic obstructive pulmonary disease. Gait Posture 2010;31(4):456-460.

3. Melzer I, Benjuya N, Kaplanski J. Postural stability in the elderly: a comparison between fallers and non-fallers. Age Ageing 2004;33(6): 602-607.

4. Piirtola M, Era P. Force platform measurements as predictors of falls among older people: a review. Gerontology 2006;52(1):1-16. 


\section{Out-Patient PR and Balance in Chronic Respiratory Disease}

5. Beauchamp MK, Hill K, Goldstein RS, Janaudis-Ferreira T, Brooks D. Impairments in balance discriminate fallers from non-fallers in COPD. Respir Med 2009;103(12):1885-1891.

6. Silva RB, Eslick GD, Duque G. Exercise for falls and fracture prevention in long term care facilities: a systematic review and metaanalysis. J Am Med Dir Assoc 2013;14(9):685-689.e2.

7. Ries AL, Bauldoff GS, Carlin BW, Casaburi R, Emery CF, Mahler DA, et al. Pulmonary rehabilitation: joint ACCP/AACVPR evidencebased clinical practice guidelines. Chest 2007;131(5 Suppl):4S-42S.

8. Beauchamp MK, Sibley KM, Lakhani B, Romano J, Mathur S, Goldstein RS, Brooks D. Impairments in systems underlying control of balance in COPD. Chest 2012;141(6):1496-1503.

9. Nield M, Hoo GS, Roper J, Santiago S, Dracup K. Usefulness of the human activity profile, a functional performance measure, in people with chronic obstructive pulmonary disease. J Cardiopulm Rehabil 2005;25(2):115-121.

10. Troosters T, Gosselink R, Decramer M. Short- and long-term effects of outpatient rehabilitation in patients with chronic obstructive pulmonary disease: a randomized trial. Am J Med 2000; 109(3):207-212.

11. Ozalevli S, Ilgin D, Narin S, Akkoclu A. Association between disease-related factors and balance and falls among the elderly with COPD: a cross-sectional study. Aging Clin Exp Res 2011; 23(5):372-377.

12. Beauchamp MK, Janaudis-Ferreira T, Parreira V, Romano JM, Woon L, Goldstein RS, Brooks D. A randomized controlled trial of balance training during pulmonary rehabilitation for individuals with COPD. Chest 2013;144(6):1803-1810.

13. Beauchamp MK, O'Hoski S, Goldstein RS, Brooks D. Effect of pulmonary rehabilitation on balance in persons with chronic obstructive pulmonary disease. Arch Phys Med Rehabil 2010;91(9): 1460-1465.

14. Collins JJ, De Luca CJ. Open-loop and closed-loop control of posture: a random-walk analysis of center-of-pressure trajectories. Exp Brain Res 1993;95(2):308-318.

15. Chang AT, Seale H, Walsh J, Brauer SG. Static balance is affected following an exercise task in chronic obstructive pulmonary disease. J Cardiopulm Rehabil Prev 2008;28(2):142-145.

16. Podsiadlo D, Richardson S. The timed "Up \& Go": a test of basic functional mobility for frail elderly persons. J Am Geriatr Soc 1991; 39(2):142-148.

17. Steffen TM, Hacker TA, Mollinger L. Age- and gender-related test performance in community-dwelling elderly people: Six-Minute Walk Test, Berg Balance Scale, Timed Up \& Go Test, and gait speeds Phys Ther 2002;82(2):128-137.

18. Dite W, Temple VA. A clinical test of stepping and change of direction to identify multiple falling older adults. Arch Phys Med Rehabil 2002;83(11):1566-1571.

19. Craig CL, Marshall AL, Sjöström M, Bauman AE, Booth ML, Ainsworth BE, et al. International physical activity questionnaire: 12country reliability and validity. Med Sci Sports Exerc 2003;35(8): 1381-1395.

20. Turnbull JC, Kersten P, Habib M, McLellan L, Mullee MA, George S. Validation of the Frenchay Activities Index in a general population aged 16 years and older. Arch Phys Med Rehabil 2000;81(8): 1034-1038.

21. Jones PW, Quirk FH, Baveystock CM, Littlejohns P. A self-complete measure of health status for chronic airflow limitation. The St.
George's Respiratory Questionnaire Am Rev Respir Dis 1992;145(6): 1321-1327.

22. Jones PW, Quirk FH, Baveystock CM. The St George's Respiratory Questionnaire. Respir Med 1991;85(Suppl B):25-31; discussion 33-27.

23. Yardley L, Beyer N, Hauer K, Kempen G, Piot-Ziegler C, Todd C. Development and initial validation of the Falls Efficacy Scale-International (FES-I). Age Ageing 2005;34(6):614-619.

24. Powell LE, Myers AM. The Activities-specific Balance Confidence (ABC) Scale. J Gerontol A Biol Sci Med Sci 1995;50A(1): M28-M34

25. Myers AM, Fletcher PC, Myers AH, Sherk W. Discriminative and evaluative properties of the Activities-specific Balance Confidence (ABC) scale. J Gerontol A Biol Sci Med Sci 1998;53(4): M287-M294.

26. ATS Committee on Proficiency Standards for Clinical Pulmonary Function Laboratories. ATS statement: guidelines for the six-minute walk test. Am J Respir Crit Care Med 2002;166(1):111-117.

27. Einstein A. Investigations on the theory of the Brownian movement. Mineola, New York: Dover Publications; 1956; page 17.

28. van Dieën JH, Koppes LL, Twisk JW. Postural sway parameters in seated balancing; their reliability and relationship with balancing performance. Gait Posture 2010;31(1):42-46.

29. Winter DA, Prince F, Frank JS, Powell C, Zabjek KF. Unified theory regarding $\mathrm{A} / \mathrm{P}$ and $\mathrm{M} / \mathrm{L}$ balance in quiet stance. $\mathrm{J}$ Neurophysiol 1996;75(6):2334-2343.

30. Winter DA, Prince F, Stergiou P, Powell C. Medial-lateral and anterior-posterior motor responses associated with centre of pressure changes in quiet standing. Neurosci Res Commun 1993;12(3):141148

31. Hodges PW, Heijnen I, Gandevia SC. Postural activity of the diaphragm is reduced in humans when respiratory demand increases. J Physiol 2001;537(Pt 3):999-1008.

32. Smith MD, Chang AT, Hodges PW. Balance recovery is compromised and trunk muscle activity is increased in chronic obstructive pulmonary disease. Gait Posture 2016;43:101-107.

33. Mok NW, Brauer SG, Hodges PW. Hip strategy for balance control in quiet standing is reduced in people with low back pain. Spine 2004;29(6):E107-E112.

34. Sattin RW, Easley KA, Wolf SL, Chen Y, Kutner MH. Reduction in fear of falling through intense tai chi exercise training in older, transitionally frail adults. J Am Geriatr Soc 2005;53(7):1168-1178.

35. Clemson L, Cumming RG, Kendig H, Swann M, Heard R, Taylor K. The effectiveness of a community-based program for reducing the incidence of falls in the elderly: a randomized trial. J Am Geriatr Soc 2004;52(9): 1487-1494.

36. Tennstedt S, Howland J, Lachman M, Peterson E, Kasten L, Jette A. A randomized, controlled trial of a group intervention to reduce fear of falling and associated activity restriction in older adults. J Gerontol B Psychol Sci Soc Sci 1998;53(6):P384-P392.

37. Rhea MR, Alvar BA, Burkett LN, Ball SD. A meta-analysis to determine the dose response for strength development. Med Sci Sports Exerc 2003;35(3):456-464.

38. Lord SR, Ward JA, Williams P, Anstey KJ. Physiological factors associated with falls in older community-dwelling women. J Am Geriatr Soc 1994;42(10):1110-1117.

39. Janaudis-Ferreira T, Wadell K, Sundelin G, Lindström B. Thigh muscle strength and endurance in patients with COPD compared with healthy controls. Respir Med 2006;100(8):1451-1457. 